\title{
Cardiac Recurrence in a Patient with Long-Term Survival from Metastatic Colon Cancer
}

\author{
Annabelle Butler Eric A. Wiebke \\ Department of Surgery, Indiana University School of Medicine, \\ Indianapolis, Ind., USA
}

\section{Key Words}

Colorectal cancer $\cdot$ Cardiac recurrence $\cdot$ Resection

\begin{abstract}
Metastatic colorectal cancer represents a major health problem in the US and worldwide. Forty percent of patients undergoing resection of the primary tumor will experience relapse. In this brief review, we describe a case of a woman with metastatic disease and long-term survival culminating with an unusual myocardial recurrence. Over three decades, a multimodality approach has evolved to allow for long-term survival in selected patients with metastatic colorectal cancer. In this case report, the role of multiple aggressive surgical resections is emphasized.
\end{abstract}

\section{Introduction}

Approximately 140,000 new cases of colorectal cancer are diagnosed annually in the United States [1]. Approximately $70 \%$ will undergo operative management and of those, about $40 \%$ will experience relapse [2]. Presented is a case study of a patient with over 17 years long-term survival of multiply recurrent metastatic colon carcinoma. In these 17 years, the patient has undergone four operations for tumor recurrence without any other adjuvant therapy besides the chemoradiation therapy completed after the time of initial diagnosis, with the latest recurrence being to the myocardium. In this case report, we wish to emphasize the integral role of surgery for recurrent disease and the unusual nature of the cardiac recurrence, and explore the implications and limitations of these multiple surgical interventions. 


\section{Case Report}

In 1992, a 60-year-old white female presented with hematochezia and change in bowel habits. Evaluation revealed rectosigmoid cancer without liver metastases on CT scan. Carcinoembryonic antigen (CEA) level was $28.7 \mathrm{ng} / \mathrm{dl}$. She underwent low anterior resection. Pathology demonstrated well-differentiated adenocarcinoma, T3N1 (stage III). The patient underwent adjuvant therapy with 5-fluorouracil, levamisole and radiation therapy, completing therapy in 1993.

In July 1995, a CT scan for persistently elevated CEA demonstrated a lesion in the left lobe of her liver. MRI confirmed the left lobe lesion and was suspicious for a right lobe lesion as well. The patient underwent partial left and partial right hepatectomies in October 1995. Surgical pathology demonstrated recurrent adenocarcinoma with negative margins in both lesions. CEA levels returned to within normal range postoperatively. In the fall of 1999, an office visit physical exam revealed a palpable mass in her old incision site from the midepigastric region to the edge of the liver. CEA levels were also slightly elevated. Subsequent CT scans confirmed local tumor recurrence. The patient underwent right rectus muscle resection with partial antrectomy for adherent tumor. Margins were negative and the patient had an uneventful recovery. In September 2006, 16 years since her initial colon cancer diagnosis, a routine CEA measurement was again slightly elevated. Repeat CT scan demonstrated areas of abnormality in what would be the falciform ligament and in the abdominal wall just right of midline. A PET scan confirmed these areas of abnormality and indicated a more intrahepatic source. The patient underwent en bloc wedge liver resection, partial diaphragm resection, partial gastrectomy and partial right chest wall resection. No surgical margins were involved and the patient had an uneventful recovery.

In April 2009, the patient underwent reevaluation for elevated CEA. The heart border was abnormal on CT scan, and transesophageal echocardiogram showed a mass in the right atrioventricular groove. MRI confirmed this mass with some tumor involvement in the right atrium. The patient was asymptomatic. Repeat CT and PET scans at this time showed no other evidence of metastatic disease ( $\underline{\text { fig. 1 }}$ ). In May 2009, the patient underwent cardiac surgery (fig. 2) and resection. The resection included right atrium and ventricular free wall with radical pericardectomy. Initial margins were involved, but were negative after additional resection. The defect was repaired with bovine pericardial patch, tricuspid valvuloplasty and bypass grafting. The patient had an uneventful recovery. A follow-up PET/CT scan 6 months after her cardiac surgery showed evidence for extension of the neoplastic disease in her left and right atria, confirmed by MRI. Gene markers confirmed tumor sensitivity to chemotherapy. The patient was started on oxaliplatin-based chemotherapy with bevacizumab. Last visit was November 2011 and she was on single agent bevacizumab without disease progression.

\section{Discussion}

Approximately 140,000 new cases of colorectal cancer are diagnosed annually in the United States [1]. According to a study of the National Cancer Institute's Surveillance, Epidemiology, and End Results (SEER) database of approximately 140,000 people with colon cancer between 1999 and 2006, the 5-year survival rate for a stage III colon cancer patient is 70\% [1]. The patient described in this case is now a 78-year-old woman with long-term survival from 17 years of recurrent colon cancer, initially stage III. She underwent low anterior resection with adjuvant chemoradiation therapy completed by 1993 and she has since undergone four more operations for metastases and local recurrences, including two liver resections, right chest wall and right abdominal wall resections, and most recently, cardiac resection. In this case, surgery was the best means of therapy for the recurrent disease at each time point and we suggest that the multiple surgical interventions are the main reason for this patient's long-term survival with metastatic colon adenocarcinoma. Table 1 shows the patient's course and interventions. 
The latest curative-intent surgery undergone by this long-term colon cancer survivor has been cardiac resection, and this has resulted in the shortest disease-free interval of all her resections ( 6 months). There appear to be a total of ten case reports in the literature since 1982 of colorectal cancer metastases to the heart [3-12]. Indeed, the literature suggests cardiac metastasis from colorectal cancer to be extremely rare, probably less than $2 \%$ of all cases based on autopsy series $[13,14]$. Currently, there are no studies investigating the role of surgery in colorectal cancer cardiac metastases. Our patient underwent one cardiac resection and at 6 months follow-up was shown to have recurrence and extension of disease on PET imaging. Chemotherapy is the treatment of choice as no further cardiac resection is being considered.

When discussing colorectal cancer survival, several contributing factors are considered. One such factor is tumor biology, such as degree of differentiation, nodal involvement at presentation, CEA level, and mutations in k-ras and p53 [15]. Although these tumor markers are not routinely tested and the exact biology of this tumor is unknown, the patient likely has a less aggressive type of colon adenocarcinoma. If this tumor were more aggressive, recurrences would likely be widely metastatic, precluding further surgical intervention. As is seen in this case, however, all of her recurrences were either isolated metastases or resectable local recurrences, deeming surgical intervention appropriate therapy.

This particular patient did not benefit from improved chemotherapy as she was diagnosed in 1992 and treated with levamisole, a drug no longer used. She did, however, benefit from surgical intervention in 1995, 1999, 2006, and 2009. The patient also received consistent follow-up with her surgeon and oncologist which included frequent CEA measurements, as well as colonoscopies, CT, MRI and PET imaging when necessary that proved essential in detecting early recurrence amenable to curativeintent salvage surgery. The interesting surgical management in this case comes not at the onset of her disease, but throughout the multiple recurrences in the past 17 years, lastly the myocardial recurrence. Surgery remained the principal clinical intervention for this patient early on and she has benefited with long-term survival. Survival for stage IV colorectal cancer has been improving dramatically since 1998 for many reasons, including improvements in chemotherapy and more aggressive surgical resections [16]. At the times of this patient's metastatic disease resections, adjuvant therapy after metastasectomy was not considered standard of care, and the issue remains controversial $[17,18]$. In the past 17 years, the patient has not undergone adjuvant chemotherapy after resection of recurrences. However, with her current cardiac recurrence, the patient underwent FOLFOX with bevacizumab and after being reevaluated for tumor sensitivity, bevacizumab only most recently. Current salvage chemotherapy regimens appear to improve survival for locally and distant recurrent disease [19].

This patient is an example of long-term metastatic colon cancer survival whose latest recurrence is in the heart, a very unusual place for colorectal metastatic disease. Surgery has been the primary intervention for her throughout her multiple recurrences. We would like to suggest that with a nonaggressive tumor biology and only locally recurrent disease, surgery can provide long-term survival for other colon cancer patients. Proper follow-up and patient selection will further ensure survival benefit from repeated surgical interventions. The role of new and active chemotherapy 
regimens in the adjuvant setting after resection of metastases remains an interesting area of future investigation.

\section{Disclosure Statement}

The authors have no conflicts of interest to report.

Table 1. Clinical course of long-term survivor with metastatic colon cancer

\begin{tabular}{llll}
\hline Date & Surgery for patient & $\begin{array}{l}\text { Type of } \\
\text { recurrence }\end{array}$ & Additional therapy received by patient \\
\hline 1992 & $\begin{array}{l}\text { 1. Low anterior resection } \\
\text { 2. Bilobar liver resections }\end{array}$ & Metastatic & 1. 5-FU/levamisole; pelvic irradiation \\
1995 & 3. En bloc abdominal wall and gastric resection & Local & \\
1999 & 4. En bloc diaphragm, stomach, liver, chest wall resection & Metastatic & 2. FOLFOX \\
2006 & 5. Cardiac resection & Local FOX + bevacizumab & 4. Bevacizumab \\
\hline
\end{tabular}

5-FU = 5-Fluorouracil; FOLFOX = 5-FU, leucovorin, oxaliplatin.

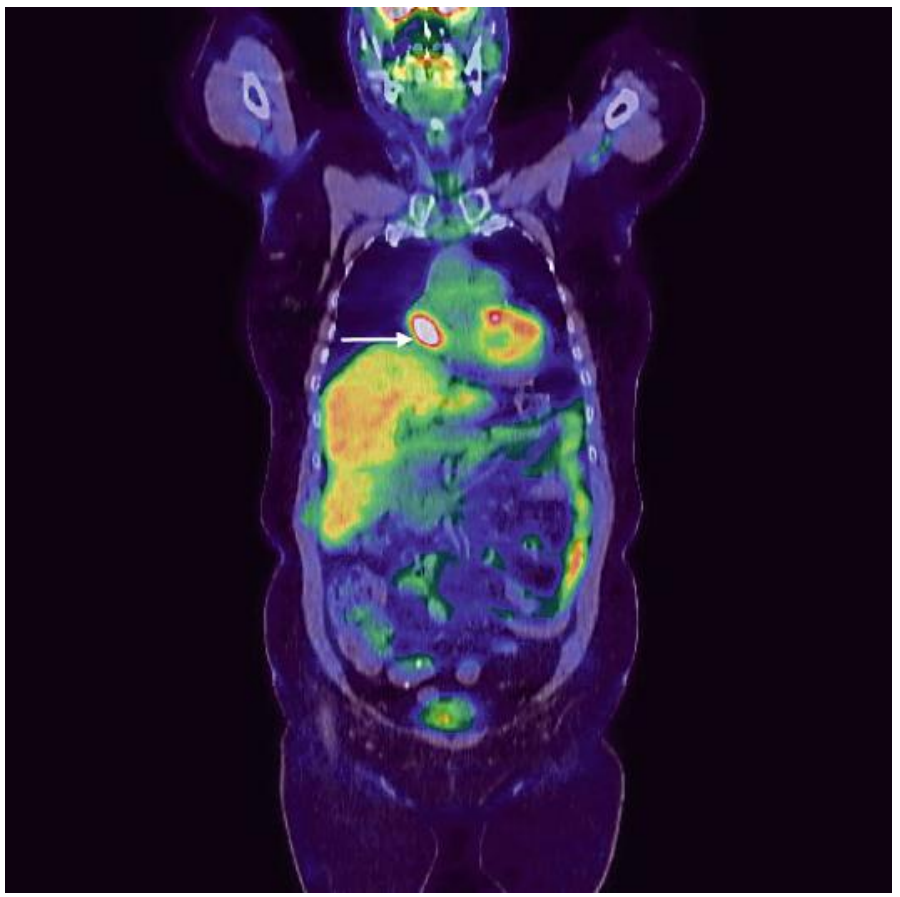

Fig. 1. PET/CT scan showing initial myocardial recurrence (white arrow). 

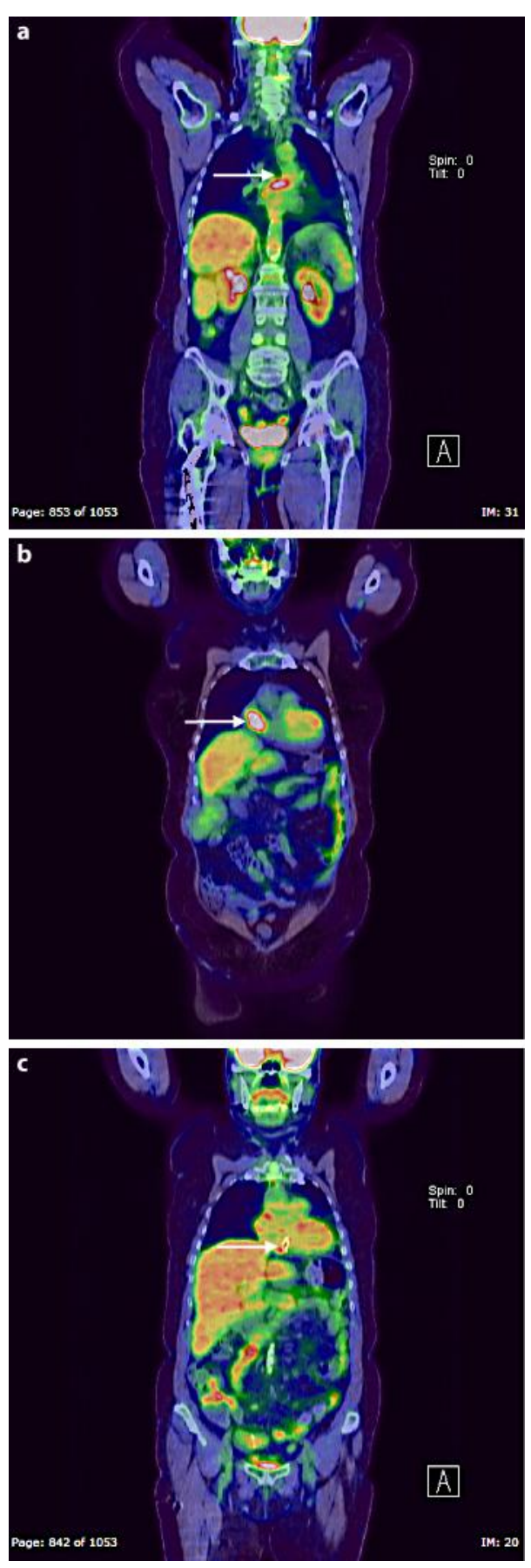

Fig. 2. PET/CT scan showing recurrence 6 months after pericardial and myocardial resection (white arrows). 


\section{References}

1 Siegel R, Ward E, Brawley 0, Jemal A: Cancer statistics, 2011: the impact of eliminating socioeconomic and racial disparities on premature cancer deaths. CA Cancer J Clin 2011;61:212-236.

-2 Bowne WB, Lee B, Wong WD, et al: Operative salvage for locoregional recurrent colon cancer after curative resection: an analysis of 100 cases. Dis Colon Rectum 2005;48:897-909.

-3 Choi PW, Kim CN, Chang SH, et al: Cardiac metastasis from colorectal cancer: a case report. World J Gastroenterol 2009;15:2675-2678.

-4 Teixeira H, Timoteo T, Marcao I: [Cardiac metastases from a colonic tumor]. Acta Med Port 1997;10:331-334.

5 Testempassi E, Takeuchi H, Fukuda Y, et al: Cardiac metastasis of colon adenocarcinoma diagnosed by magnetic resonance imaging. Acta Cardiol 1994;49:191-196.

-6 Lord RV, Tie H, Tran D, Thorburn CW: Cardiac metastasis from a rectal adenocarcinoma. Clin Cardiol 1999;22:749.

7 Parravicini R, Fahim NA, Cocconcelli F, et al: Cardiac metastasis of rectal adenocarcinoma. Surgical treatment. Tex Heart Inst J 1993;20:296-298.

8 Oneglia C, Negri A, Bonora-Ottoni D, et al: Congestive heart failure secondary to right ventricular metastasis of colon cancer. A case report and review of the literature. Ital Heart J 2005;6:778-781.

19 Chu PH, Ko YL, Liao WB, Chiang CW: Metastatic colonic carcinoma with intracavitary right ventricular outflow tract obstruction and cardiac tamponade: a case report. Changgeng Yi Xue Za Zhi 1996;19:264267.

10 Koizumi J, Agematsu K, Ohkado A, et al: Solitary cardiac metastasis of rectal adenocarcinoma. Jpn J Thorac Cardiovasc Surg 2003;51:330-332.

11 Case records of the Massachusetts General Hospital. Weekly clinicopathological exercises. Case 45-1992. A 75-year-old man with carcinoma of the colon and a right ventricular mass. N Engl J Med 1992;327:1442-1448.

12 Henuzet C, Franken P, Polis O, Fievez M: Cardiac metastasis of rectal adenocarcinoma diagnosed by twodimensional echocardiography. Am Heart J 1982;104:637-638.

13 Klatt EC, Heitz DR: Cardiac metastases. Cancer 1990;65:1456-1459.

14 Mukai K, Shinkai T, Tominaga K, Shimosato Y: The incidence of secondary tumors of the heart and pericardium: a 10-year study. Jpn J Clin Oncol 1988;18:195-201.

15 Kulesz-Martin M: Molecular markers for colon cancer: where do we go from here? Cancer Invest 2000;18:287-288.

16 Kopetz S, Chang GJ, Overman MJ, et al: Improved survival in metastatic colorectal cancer is associated with adoption of hepatic resection and improved chemotherapy. J Clin Oncol 2009;27:3677-3683.

17 Ruo L, DeMatteo RP, Blumgart LH: The role of adjuvant therapy after liver resection for colorectal cancer metastases. Clin Colorectal Cancer 2001;1:154-166; discussion 167-158.

18 Parks R, Gonen M, Kemeny N, et al: Adjuvant chemotherapy improves survival after resection of hepatic colorectal metastases: analysis of data from two continents. J Am Coll Surg 2007;204:753-761; discussion 761-753.

19 Tol J, Koopman M, Cats A, et al: Chemotherapy, bevacizumab, and cetuximab in metastatic colorectal cancer. N Engl J Med 2009;360:563-572. 